\title{
Design of Single Prism Coded Aperture Snapshot Spectral Imager using Ray Tracing Simulation
}

\author{
Mengjia Ding ${ }^{* \dagger}$, Peter WT Yuen ${ }^{* \ddagger}$, Mark A Richardson *§ \\ * Centre for Electronic Warfare, Information and Cyber \\ Cranfield University, Defence Academy of the United Kingdom \\ Shrivenham, United Kingdom \\ $\dagger$ m.ding@cranfield.ac.uk; ${ }^{\ddagger}$ p.yuen@cranfield.ac.uk; $§$ m.a.richardson@cranfield.ac.uk
}

\begin{abstract}
Novel imaging systems published in the literature mostly concern with the performance of the final stage of the designed system which normally accompanies with a brief description of the system configuration only. Other information, such as how the system was optimized and the methodology adopted for improving them to their final stage are heavily lacking in the open domain. This paper addresses this issue by providing a guide for the modeling of compressive imaging based on Single Disperser Coded Aperture Snapshot Spectral Imaging (SD-CASSI), with focuses on the optimization of the dispersion capability, the reduction of spatial and chromatic aberrations for enhancing the performance of the SD-CASSI. As an example the system is designed for a numerical aperture of $0.125,0.3 \%$ distortion at central wavelength $587.56 \mathrm{~nm}$, and 32 spectral bands with a spatial resolution of $13 \mu \mathrm{m}$. The system was simulated by ray tracing program TracePro.
\end{abstract}

Index Terms - computational imaging, ray tracing, optical system design, CASSI, TracePro, OSLO

\section{INTRODUCTION}

Computational imaging [1]-[4] is a process for reproducing the image of the scene through multidimensional model of sensing and compuationally reconstruction of the scene. One good example is the compressive imaging based Coded Aperture Snapshot Spectral Imager (CASSI) which was firstly published by [5] and later a variation of enhanced prototypes have been reported [6]-[10]. The CASSI features low system cost, efficient image data requisitions without scanning [4]. The CASSI system is technologically fascinating and the present paper focuses on how one can design the system and to optimize its performance for a given system configuration and optical components. As an example the paper adopted the Single Disperser CASSI (SD-CASSI) configuration with a single prism as the dispersion element. The layout of the paper consists of two parts: (a) Design procedures for the Single Prism CASSI (SP-CASSI) using optical design software OSLO to optimize the rotation angle of single equilateral prism for the reduction of chromatic aberration. (b) The ray tracing simulation of the designed system by TracePro under synthetic illumination environment for the imaging of scene which consists of colored objects. The scene is reconstructed from the multiplexed spectral spatial data using Two-Step Iterative Shrinkage/Thresholding (TwIST) decompressive algorithm [11].

\section{Coded Aperture SnApshot Spectral IMAGer}

\section{A. System Model}

CASSI principle relies on spatial modulator and spectral modulator. Concretely speaking, according to [12], spectral function of incident light at the entrance of system can be represented as $S_{0}(x, y, l)$ where $(x, y)$ denotes two spatial dimension and $l$ denotes one spectral dimension. The spatial modulation, implemented by a two-dimensional physical mask of coded pattern as $M(x, y)$, is

$$
S_{1}(x, y, l)=S_{0}(x, y, l) M(x, y) .
$$

Spectral modulation was implemented by a dispersive prism to generate one-spatial-dimensional spectral dispersion, where the dispersed deviation depends on the prism's dispersion ability as $D(l)$. If we locate the central wavelength $l_{c}$ to calculate the relative dispersion, the spectral function after the spectral modulation is

$$
S_{2}(x, y, l)=S_{1}\left(x, y+D\left(l-l_{c}\right), l\right) .
$$

Therefore, the measurement at detector is the integration of the light from interested region and interested wavelengths as follows,

$g=\iiint S_{0}\left(x, y+D\left(l-l_{c}\right), l\right) M\left(x, y+D\left(l-l_{c}\right)\right) d x d y d l$.

\section{B. Reconstruction Algorithm}

SP-CASSI measured a two-dimensional multiplex data, which can be solved using unconstrained optimization as

$$
\hat{S}(t, \mathrm{R})=\underset{S}{\arg \min }\left[\frac{1}{2}\|g-H S\|_{2}^{2}+t \mathrm{R}(S)\right],
$$

where $H$ is the measurement matrix that contains spatial modulation and spectral modulation. $\hat{S}$ is the estimate data cube constrained by the tuning parameter $t$ and the regularizer $\mathrm{R}(S)$. The regularizer $\mathrm{R}$ in this work selected Total Variation (TV) [13] regularization to regularize the estimate data cube in two spatial dimensions. TwIST was introduced to find the solution in (4). 


\section{System DESIGN}

SP-CASSI consists of an objective lens, two imaging lenses, and an equilateral prism. It is common to begin with offthe-shelf optics as the backbone of the entire system. In this work, we used a patent US7050245B2 [14] as the basic lens to construct the system. This lens assembly has a $25 \mathrm{~mm}$ focal length at a full Field-of-View (FoV) of $15^{\circ}$ with $0 \%$ vignetting and less than $1 \%$ distortion, which provides an excellent start point.

\section{A. Resolution}

All the considerations of system resolution are calculated based upon two $6.5 \mu \mathrm{m}$ sensor pixels referred to our lab detector. The required system resolution, therefore, by applying Nyquist sampling rate $\frac{1}{2}$ (line pair)/mm per pixel, gives

$$
\frac{1}{2 \times 0.013 \mathrm{~mm}}=38.46 \mathrm{lp} / \mathrm{mm} \text {. }
$$

System F-number under such resolution, which satisfies the Rayleigh criterion 9\% MTF would be F/32 over the required spectral range from $450 \mathrm{~nm}$ to $650 \mathrm{~nm}$, so that it requests an F-number, i.e. F/16 or faster to ensure $50 \%$ MTF at spatial frequency $38.46 \mathrm{lp} / \mathrm{mm}$. The aforementioned lens assembly could be set to F/4 to allow the system much tolerance by adjusting the aperture radius of lens elements and the aperture stop, which provides the numerical aperture 0.125 . This $F / 4$ system yields $44 \mathrm{lp} / \mathrm{mm}$ at $450 \mathrm{~nm}, 160 \mathrm{lp} / \mathrm{mm}$ at $587 \mathrm{~nm}$, and $80 \mathrm{lp} / \mathrm{mm}$ at $650 \mathrm{~nm}$, under monochromatic MTF of $50 \%$.

\section{B. Coded Aperture}

Pattern of coded aperture was generated by Matlab in Fig. 1 using a random sequence in a $4 \times 4$ matrix with $50 \%$ of zeros and $50 \%$ of ones, partitioned from a $128 \times 128$ pattern matrix and shuffled 32 times to get the final pattern, where the zero represents square closed hole and the one represents square open hole. This partition method is to prevent consecutive zeros area in the pattern from being too large to reconstruct image. The size of pattern was designed in $13 \mu \mathrm{m}$ per square hole that is 2 times detector pitch, resulting in a $1.664 \mathrm{~mm} \times 1.664 \mathrm{~mm}$ pattern. Such size is due to, one hand, it is enough for the purpose of demonstrating system performance; the other hand, balancing the consumed

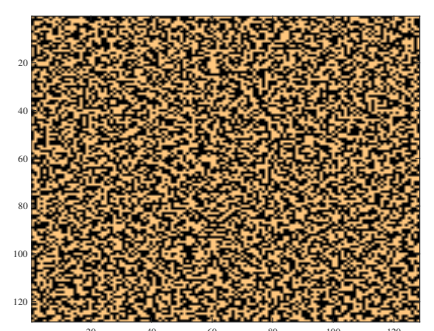

Fig. 1. $128 \times 128$ Coded Aperture pattern is displayed in Matlab, which was created by randomly shuffling $4 \times 4$ sub-matrix 32 times and modeled in TracePro with a size of $1.664 \mathrm{~mm} \times 1.664 \mathrm{~mm}$.
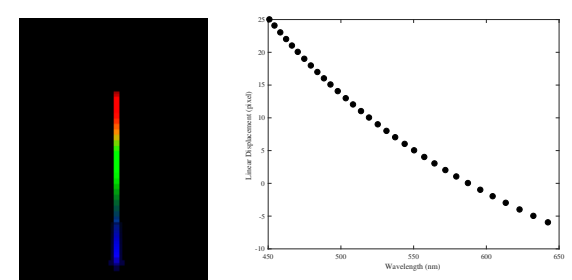

Fig. 2. Dispersion displacement of system response to single pixel source $13 \mu \mathrm{m} \times 13 \mu \mathrm{m}$. The left side of picture shows the simulated result in RGB color in TracePro. The $\mathrm{x}$-axis denotes wavelength in $\mathrm{nm}$ and the $\mathrm{y}$-axis denotes linear displacement in pixel.

computer resource for ray tracing, that large object to simulate could yield tremendous rays to encounter the entire coverage of the object and cost plenty of processing time. Such size of coded aperture (half size), or equivalently $0.832 \mathrm{~mm}$ object height of imaging lens, would yield an field angle $1.906^{\circ}$ paraxially approximated as

$$
\text { Image Height }=E F L \cdot \tan (\text { Field Angle }),
$$

where EFL denotes Effective Focal Length. Image Height represents the object height of imaging lens due to the reversed placement of the imaging lens.

\section{Single Prism Dispersion}

The dispersive element was chosen from a common equilateral prism BK-7 with $16 \mathrm{~mm}$ wide that accounts for the size of lens assembly in which the largest aperture radius has only $5 \mathrm{~mm}$. A rotation angle $49.45^{\circ}$ by a non-perpendicular position between the prism and system axis to generate dispersion was optimized to yield a approximate 1:1 matched image size. Concretely, when a coded-aperture-size object $(-0.832 \mathrm{~mm}$ to $+0.832 \mathrm{~mm}$ ) is under monochromatic wavelength $587.56 \mathrm{~nm}$ illumination, this rotation angle corresponding to the incident angle that encounters the first surface of prism, results in an optimal image height from $-0.8537 \mathrm{~mm}$ to $+0.8053 \mathrm{~mm}$, which provides a distortion of $0.3 \%$ compared to the $1.664 \mathrm{~mm}$ object. Likewise, to the short wavelength $450 \mathrm{~nm}$ and long wavelength $650 \mathrm{~nm}$, it yields a distortion of $0.9 \%$ and $0.6 \%$, respectively, which is equivalently almost two detector pixels $(13 \mu \mathrm{m})$.

More importantly, on-axis single point source of $450 \mathrm{~nm}$ to $650 \mathrm{~nm}$ would be dispersed by the prism with a range of angular deviation from $-0.75^{\circ}$ to $0.2^{\circ}$ referred to the central wavelength, corresponding to the dispersion displacement at image plane of the back imaging lens from $-0.325 \mathrm{~mm}$ to $0.078 \mathrm{~mm}$. Based on that, a set of wavelengths can be selected to form a group of linear dispersed displacement with sampling pitch $13 \mu \mathrm{m}$, resulting in 32-band measurement matrix for reconstruction. The dispersion results in Fig. 2 shows the simulation result of dispersed displacement of those 32 bands selected from $450 \mathrm{~nm}$ to $650 \mathrm{~nm}$ in vertical direction and a pixelated displacement as a function of wavelength calculated in Matlab. 


\section{System Simulation}

Optical ray tracing program TracePro is capable of simulating the interaction between geometrical rays and solid models in three dimensional environment such as absorption, reflection, and refraction by using "Generalized Ray Tracing". The solid model of the designed system is aided by solid modeling engine ACIS $^{\circledR}$ [15], depicted in silhouettes mode in Fig. 3. How to simulate the capture of synthetic targets in a synthetic environment by SP-CASSI will be explained as follows.

\section{A. Ray tracing strategy}

In order to achieve a synthetic environment, two points for illumination setup need to be considered before conducting ray tracing simulation: (a) Due to the fact that objective lens aims at the collimated rays from object at infinity (or the distance is far enough), object at infinity could lead to an infinite large size, which cannot be achieved in simulation. The objective lens, therefore, was simplified to be a single plane, or called second principal plane, which all incident rays would all go through; thus tracing those rays becoming possible. (b) Despite infinite rays forming a image in real world, it was exploited that reverse tracing the rays that form a $256 \times 256$ pixel image converging to a finite number of sampling points at second principal plane of the objective lens. In other word, each sampling point is a divergent point source of a $256 \times 256$ pixel image light source. Setting up 25 sampling point uniformly at the plane represents each one of pixels on the image was focused on by 25 rays and this way is essential to satisfy the visual requirement of image quality.

\section{B. Synthetic targets}

Four artificial targets for the simulation, character "C", " $\mathrm{R}$ ", "U", and "N" as depicted in Fig. 4, were limited within an area of $1.664 \mathrm{~mm} \times 1.664 \mathrm{~mm}$, as entry source propagating through the system. Other than directly tracing emitting rays from targets itself, the target source was collected from the reflected rays by targets under $256 \times 256 \times 32$ light source converging at those 25 sampling points at second principal plane and continuing to strike the targets, and reverse-illuminated the collected rays onto the coded aperture. Such way was able to project a focused image of targets onto the plane where coded aperture without tracing ray source from the targets at infinity. The spectral characteristics of targets were applied with four

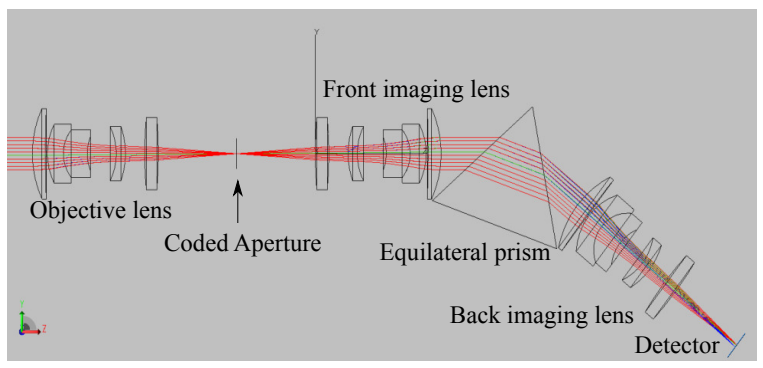

Fig. 3. Overview of SP-CASSI modeled in silhouettes mode in TracePro.

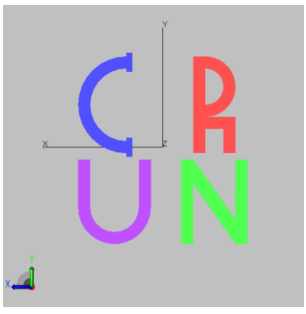

Fig. 4. Four artificial targets modeled in Trace were limited to match the scale of coded aperture, applied with distinguished custom spectral characteristics. Displayed colors of targets are only for visualization.

distinguished custom properties, representing blue, red, purple, and green color, respectively.

\section{Simulation Results and Discussion}

As mentioned in Sec. II, the reconstruction algorithm requires two inputs to form measurement matrix $\mathrm{H}$ : the coded pattern to spatially modulate incident data and the dispersion function as a function of wavelength to spectrally modulate spatially-coded data. Instead of measuring them separately, illuminating a uniform light source of 32 calibrated wavelengths onto the coded aperture would achieve both of them. Those 32 individual calibrated frames were trimmed and normalized to form a measurement matrix for further reconstruction. In Fig. 5, a cropped multiplex measurement was taken by a detector of $512 \times 512$ pixels with $6.5 \mu \mathrm{m}$ pitch in TracePro and processed to a $256 \times 318$ pixels input entry in Matlab. From the measurement, it can be noticed that a multiplex effect occurred very clear in character " $U$ " due to the applied property that has a peak response in both blue zone and red zone, as a consequence of an overlapped shaded pattern. Furthermore, other than aligned locations of character " $C$ " and character "R" set in Fig. 4, an unaligned result between them was marked due to the dispersion displacement of each peak response region. The reconstructed 32 spectral frames after running TwIST for 100 iterations.

Reconstruction result in Fig. 6 shows the spatial recovery at 32 individual wavelengths, generated in TwIST tuning parameter $t$ set to 0.05 with 100 times iteration. The condition of spatial recovery is highly matched with the targets. More

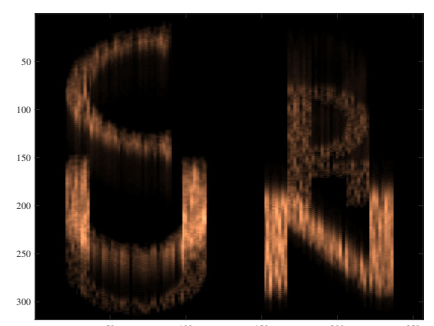

Fig. 5. Detector measurement collected from TracePro were cropped to an image of $256 \times 318$ pixels displayed in Matlab. The raw measurement were pixelated by a $512 \times 512$ detector with $13 \mu \mathrm{m}$ pitch. 

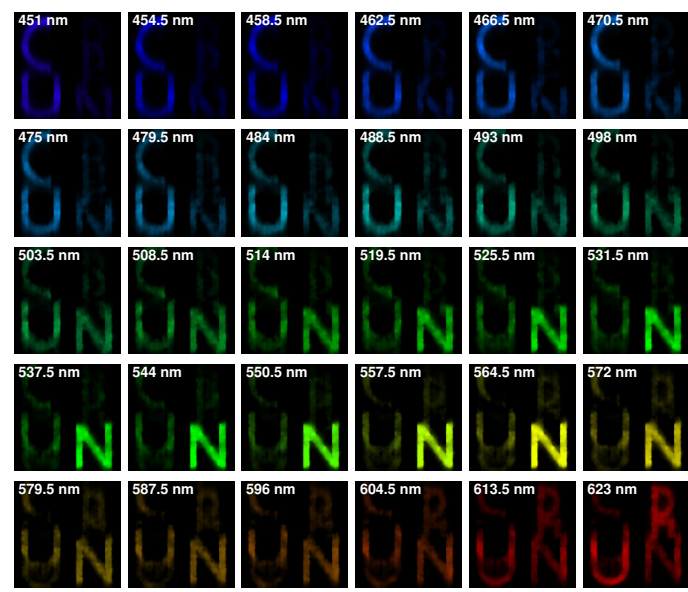

$604.5 \mathrm{~nm}$
5
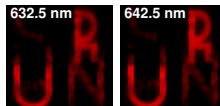

Fig. 6. Reconstruction results of 32 frames generated by TwIST with tuning parameter $t=0.05$ and 100 iterations.

importantly, the spectral recovery of each target in Fig. 7 was in comparison with the ground truth of reference under normalized ratio. The Region of Interest (ROI) selected to plot a spectral estimation curve was sampled in an area of $10 \times 10$ pixels on each targets. Despite that the reconstruction of " $U$ " has an average $20 \%$ offset at spectral region from $520 \mathrm{~nm}$ to $570 \mathrm{~nm}$, which could be due to the strong overlapping in raw data, other spectral targets with single spectral response peak are well reconstructed. That proves the designed system is able to identify spectral objects in simulation.

\section{CONCLUSION}

The paper provides a guide for the computational design of the single prism coded aperture snapshot spectral imager (SP-CASSI) using lens design software OSLO and ray tracing TracePro. The system is modeled for spectral resolvable ability of 32 bands from $450 \mathrm{~nm}$ to $650 \mathrm{~nm}$ range and spatial resolution of $13 \mu \mathrm{m}$ per band with an FoV of $3.8^{\circ}$. The system is ray traced under 32-band of homogeneous light source of $256 \times 256$ rays per band emitting from 25 sampling directions. The simulation takes about 45 minutes for ray tracing one frame. The image reconstruction (de-multiplexing) based on one frame measurement processed by the TwIST algorithm exhibits satisfactory result comparable to that of SD-CASSI reported in [5].

\section{REFERENCES}

[1] M. Levoy, "Light fields and computational imaging," IEEE Computer, vol. 39, pp. 46-55, Aug. 2006.

[2] B. Sun et al., "3D computational imaging with single-pixel detectors," Sci., vol. 340, no. 6134, pp. 844-847, 2013.

[3] T. Fromenteze et al., "Computational imaging using a mode-mixing cavity at microwave frequencies," Appl. Phys. Lett., vol. 106, no. 19, p. 194104, 2015.
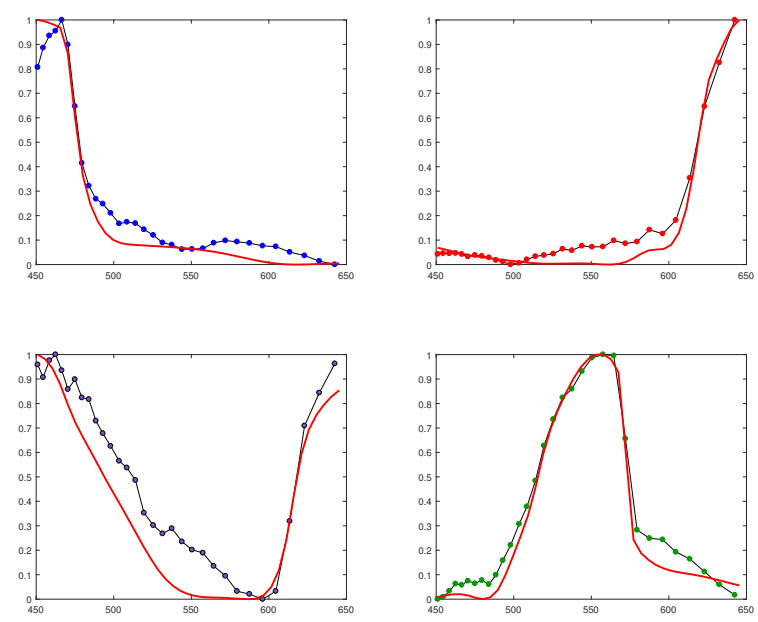

Fig. 7. Reconstruction results of spectral characteristics in normalized reflectance of individual targets "C"(upper left), "R"(upper right), "U"(lower left), and " $\mathrm{N}$ "(lower right), in comparison with the reference shown in red solid line. The $\mathrm{x}$-axis denotes wavelength in $\mathrm{nm}$ and the $\mathrm{y}$-axis denotes normalized reflectance.

[4] X. Cao et al., "Computational snapshot multispectral cameras: toward dynamic capture of the spectral world," IEEE Trans. Image Process., vol. 33, no. 5, pp. 95-108, 2016.

[5] A. Wagadarikar, R. John, R. Willett, and D. J. Brady, "Single disperser design for coded aperture snapshot spectral imaging," Appl. Opt., vol. 47, no. 10, pp. B44-B51, Apr. 2008.

[6] X. Cao, H. Du, X. Tong, Q. Dai, and S. Lin, "A prism-mask system for multispectral video acquisition," IEEE Trans. Pattern Anal. Mach. Intell., vol. 33, no. 12, pp. 2423-2435, Dec. 2011.

[7] D. S. Kittle, D. L. Marks, and D. J. Brady, "Design and fabrication of an ultraviolet-visible coded aperture snapshot spectral imager," Opt. Eng., vol. 51, no. 7, p. 071403, May 2012.

[8] T. Tsai and D. J. Brady, "Coded aperture snapshot spectral polarization imaging," Appl. Opt., vol. 52, no. 10, pp. 2153-2161, Apr. 2013.

[9] C. V. Correa, H. Arguello, and G. R. Arce, "Snapshot colored compressive spectral imager," J. Opt. Soc. Am. A, vol. 32, no. 10, pp. 1754-1763, Oct. 2015.

[10] X. Wang, Y. Zhang, X. Ma, T. Xu, and G. R. Arce, "Compressive spectral imaging system based on liquid crystal tunable filter," Opt. Express, vol. 26, no. 19, pp. 25 226-25 243, Sep. 2018.

[11] J. M. Bioucas-Dias and M. A. T. Figueiredo, "A new TwIST: two-step iterative shrinkage/thresholding algorithms for image restoration," IEEE Trans. Image Process., vol. 16, no. 12, pp. 2992-3004, Dec. 2007.

[12] G. R. Arce, D. J. Brady, L. Carin, H. Arguello, and D. S. Kittle, "Compressive coded aperture spectral imaging: An introduction," IEEE Signal Process. Mag., vol. 31, no. 1, pp. 105-115, Jan. 2014.

[13] A. Chambolle, "An algorithm for total variation minimization and applications," J. Math. Imaging Vis., vol. 20, no. 1-2, pp. 89-97, Jan. 2004.

[14] J. Tesar and R. L. Thompson, "Lens assembly and optical imaging using same," U.S. Patent 7050 245B2, May 16, 2004.

[15] TracePro User's Manual, Lambda Research Corp., Littleton, MA, 2016, release 7.7. 